\title{
A novel prognostic signature for colorectal cancer based on seven-metabolic-related genes
}

\section{Wenjing Zhang}

Medical School, Kunming University of Science and Technology, China

Dongmei Li

Medical School, Kunming University of Science and Technology, China

Mei Chen

Medical School, Kunming University of Science and Technology, China

Xiaoli Chen

Medical School, Kunming University of Science and Technology, China

Yu Zhang ( $\nabla$ yuzhang320@sina.com )

First People's Hospital of Yunnan Province

\section{Research Article}

Keywords:

Posted Date: February 23rd, 2022

DOI: https://doi.org/10.21203/rs.3.rs-1321138/v1

License: (c) (1) This work is licensed under a Creative Commons Attribution 4.0 International License. Read Full License 


\section{Abstract}

Emerging studies have suggested that metabolic enzymes and transporters (METs) have a close relationship with cancer development. However, the prognostic value and the associated mechanisms of METs in colorectal cancer (CRC) remain unclear. In this study, our aim was to identify prognostic MET signature and elucidate the potential underlying mechanism of MET in regulating CRC process. A total of 326 differential expression of MET genes (DEMETs) were identified by Limma R package with data downloaded from TCGA and GEO databases. Univariate and multivariate Cox regressions identified GDPD3, AQP8, GPX3, HPGD, CKMT2, CPT2 and CLCA1 as a robust prognostic METs signature, followed by the construction of the risk score model and nomogram in predicting the prognosis of CRC patient. TIDE algorithm showed that CRC patients in low- and high-risk groups had different response to immunotherapy. Somatic processes were downloaded from the GDC database and found that MUC16, FAT4, BDP1, CMYA5, ZNF735, CENPE, SLITRK2, ZNF208, CFAP46 and ALDH1A2 were the top 10 mutated genes in both the high- and low-risk groups. Moreover, a pharmacological network including HPGD, 38 active compounds and 115 herbs was constructed. These findings enrich our understanding of the relationships among METs, immune, and CRC, and may provide novel ideas in the treatment of CRC patients.

\section{Introduction}

Colorectal cancer (CRC) is the second most commonly diagnosed cancer in China as reported in $2019^{1}$. Depending on the symptoms, the number and size of liver metastasis, primary tumor location, the status of gene mutation and the general condition, patients may need to undergo surgery, chemotherapy, radiation therapy, targeted therapy and treatment of immune checkpoint inhibitors ${ }^{2}$. Although the combination of the above strategies has brought many clinical benefits to patients, about more than $60 \%$ of CRC patients were firstly diagnosed as metastatic CRC ( $m C R C)$ accompanied with liver or lung metastasis ${ }^{3}$ Therefore, the development of novel biomarkers in CRC may provide more benefits in early diagnosis and guidance in properly choosing therapy and monitoring of recurrence.

Abnormal metabolism is a hallmark of cancer biology ${ }^{4,5}$. It directly regulates a variety of cellular activities, including cell proliferation, tumor progression, invasion and drug resistance. For example, glutamine plays an important role in cancer cell proliferation through supplying carbon and nitrogen for nucleotide biosynthesis ${ }^{6}$. Glucose metabolism is another main source to provide metabolic carbon in cancer cells ${ }^{7}$. Glutamine and glycolysis metabolisms promote invasion and metastasis by regulating the acid value of the tumor microenvironment and lead to metalloprotease gene transcription ${ }^{8}$. Those above processes are regulated by some oncogenic or tumor- suppressive signalings, such as MYC, HIF-1, AMPK, $\mathrm{p} 53^{9-12}$. Some certain metabolism-targeting drugs could inhibit key metabolic step, including glycolysis, $\mathrm{NAD}^{+}$regeneration, fatty acid synthesis and glutaminolysis ${ }^{13}$. Furthermore, cancer metabolism is associated with the immune microenvironment and antitumor immune responses. For instance, increased lactate levels not only attenuate dendritic and T cell activation and monocyte migration, but 
also stimulates the polarization of resident macrophages to M2 state, which involved in immunosuppression ${ }^{14}$. Although metabolic enzymes and transporters (METs) represent a group of important players in cancer development, its prognostic value and potential molecular mechanisms underlying $\mathrm{CRC}$ remain to be elucidated.

With the rapid development of bioinformatics, a great number of novel cancer biomarkers have been explored for diagnosis and prognosis of tumors based on publicly available databases. Due to the high heterogeneity of tumor, using a gene set to construct a prognostic signature would be better than a single gene pattern. In the current study, a metabolism-based prognostic gene signature containing seven MET genes, was analyzed and verified using data from TCGA and Gene Expression Omnibus (GEO) datasets. Patients with CRC were divided into high- and low-risk score groups according to the signature. Additionally, the significant difference in the distribution of immune cells and gene mutation between the high- and low-risk groups according to this metabolism-based prognostic signature were further observed. This study enriches our understanding of the relationships among MET, immune and CRC, and may provide potential biomarkers for the diagnosis, prognosis and new therapeutic targets of CRC.

\section{Results}

Identification of 326 DEMETs in CRC. To identify differentially expressed metabolic enzymes and transporters (DEMETs) and analyze their association with prognosis and tumor biology in CRC, we performed an integrated bioinformatics analysis as seen in Fig. 1. A total of 1,737 differentially expressed genes (DEGs) were firstly identified between CRC tissues and control samples, which includes 780 upregulated and 957 down-regulated DEGs in CRC in relative to controls (Fig. 2A). By overlapping 1,737 DEGs with 2,752 METs derived from the comprehensive gene list of human METs, we obtained 326 DEMETs in CRC (Fig. 2B). To investigate the role of DEMETs in CRC, we performed KEGG and GO enrichment analyses. DEMETs were significantly enriched into $50 \mathrm{KEGG}$ pathways. Top10 pathways were mainly involved in metabolic pathways, as shown in Fig. 2C, such as drug metabolism - other enzymes, purine metabolism, retinol metabolism, drug metabolism - cytochrome P450, fatty acid degradation, steroid hormone biosynthesis and fatty acid metabolism (Fig. 2C). A total of $680 \mathrm{GO}$ terms were significantly enriched, and we found that the DEMETs were mainly involved in biological processes related to metabolism and transport, such as anion transmembrane transport, organic anion transport, organic acid transmembrane transport, carboxylic acid transmembrane transport, carboxylic acid transport, organic acid transport, small molecule catabolic process, alcohol metabolic process, steroid metabolic process, cofactor metabolic process and so on (Fig. 2D). Top15 enrichment GO terms of molecular function were shown in Fig. 2E, including anion transmembrane transporter activity, active transmembrane transporter activity, and metal ion transmembrane transporter activity, and so on.

Construction of METs prognostic model for CRC. From the prognostic perspective, we analyzed the 326 DEMETs in the training dataset of TCGA-CRC cohort. As a result, nine METs were selected as most significant prognostic genes, including GDPD3, AQP8, GPX3, HPGD, ADH1B, CKMT2, CPT2, CLCA1 and PPAP2A, by a univariate Cox regression $(p<0.05)$. To further identify METs with highest predictive value 
of $\mathrm{CRC}$, these nine METs were subjected to a multivariate Cox regression. It showed seven out of the nine METs comprised of GDPD3, AQP8, GPX3, HPGD, CKMT2, CPT2 and CLCA1, had robust association with prognosis of CRC patients in the training set $(p<0.05)$ (Table 1$)$.

Thereafter, a genetic risk model was established to calculate risk score of each patient in the training set, to evaluate the prognostic value of this METs signature of seven genes. Based on the median of the risk score, the CRC patients in the training set were divided into high- and low-risk groups. Kaplan-meier analysis showed that patients in low-risk group had better survival rate than patients in the high-risk group $(P<0.01$, Fig. 3A). Consistent result was observed when the validation set of TCGA-CRC cohort was subjected to survival analysis (Fig. 3B). To verify whether the 7-gene METs signature presented similar predictive value which can be extended to other CRC cohorts, a repeated analysis was applied to generate the risk score of each CRC patient collected in GSE39528 database. Consistently, it was observed that CRC patients of GSE39528 database in low-risk group had better overall survival tendency in comparison with patients in the high-risk group ( $P<0.0001$, Fig. 3C). The areas under the ROC curves for 1-, 2- and 3year were $0.633,0.632$ and 0.632 , indicating a good performance of this risk model in the training set (Fig. 3D).

To further confirm the prognostic potential of this genetic risk score model, we combined all patients from the training set and testing set together, and performed univariate and multivariate analyses on the combined dataset to detect independent prognostic factors of CRC (Table 2). By univariate analysis, only gender was excluded since it was not associated with prognosis of CRC (HR 0.965, $P=0.86)$. In the further multivariate analysis, risk of the 7-gene MET signature exhibited a significant relationship with prognosis of CRC (HR 1.79, $P=0.009)$, as well as other clinical parameters such as age, TNM stage, indicating the predictive value of the 7-gene MET signature as independent prognostic factor of CRC. Furthermore, we established a prognostic nomogram for OS of CRC patients. As shown in Fig. 3E, the nomogram predicting 1-, 3-, and 5-year OS was built on the basis of the chosen parameters with significant difference based on the above multivariate Cox analysis. The nonogram showed the greatest contributing factor to prognosis was the age, followed by T, N, M and high-risk group. Through adding all these values and placing them into the total-point scale, the survival probabilities were calculated. The correction curve was then drawn based on the above prediction model. The C-index of this nomogram indicating OS, was 0.7729 . The calibration plots presented an optimal agreement between the outcomes predicted by the nomogram and those actually observed in terms of the 1-, 3-, and 5-year OS. After calculation, the slope of $1-, 3$ - and 5 -year is $0.7936,0.5742$ and 0.3453 respectively, indicating that the prediction effect is the best at 1-year (Fig. 3F).

GSEA revealed immune-related gene sets between high- and low-risk groups of METs signature. To better understand the underlying mechanisms of prognostic METs biomarkers in regulating CRC development, we analyzed the dysregulated genes between high- and low-risk groups by Gene Set Enrichment Analysis (GSEA), and found a total of 82 KEGG pathways and $2092 \mathrm{GO}$ terms were significantly enriched. Among the top 10 KEGG pathways, except peroxisome pathway, cell adhesion molecules CAMs, complement and coagulation cascades, cytokine/cytokine receptor interaction, ECM receptor interaction, focal adhesion, 
graft versus host disease, system lupus erythematosus and viral myocarditis were all upregulated (Fig. 4A). As for GO terms ((Fig. 4B), some immune-related gene sets were enriched, such as adaptive immune response, adaptive immune response based on somatic recombination of immune receptors built from immunoglobulin superfamily domains, blood microparticle, indicating that immune plays an important role in prognostic MET regulated CRC.

\section{Different tumor immune microenvironment between low- and high-risk groups. Based on the GSEA} analysis, we hypothesized that the tumor immune microenvironment of low- and high-risk groups was different. Thus, we analyzed the immune infiltration between two groups by sSGSEA. Except activated CD4 T cells, activated CD8 T cells, CD56bright natural killer cells, effector memory CD4 T cells, immature dendritic cells, monocytes, neutrophils, type $17 \mathrm{~T}$ helper cells and type $2 \mathrm{~T}$ helper cells, 19 immune cells were significantly infiltrated into low- and high-risk groups (Fig. 5A). Moreover, we found that the expressions of prognostic MET biomarkers were strongly correlated with at least the abundance of three immune cell types (Fig. 5B), further suggesting the close relationship between MET and immune. In addition, the expressions of CD27, CD274, HAVCR2, LAG3, PDCD1LG2 and TIGIT were remarkably different between low- and high-risk groups (Fig. 5C). All of the above results demonstrated that the immune microenvironment was different between low- and high-risk groups, which may lead to different immunotherapeutic response. Therefore, TIDE was applied to predict the response of two groups to immunotherapy, and indeed different TIDE scores were observed (Fig. 5D). Due to immune checkpoint inhibitors targeting PD-1/PD-L1/CTLA4 are hot topics in the treatment of advanced malignant tumors, we also used SubMap algorithm to predict the likelihood of different subtype responding to immunotherapy. As shown in Fig. 5E, patients in high-risk group seems to be more sensitive to the treatment of CTLA4 inhibitors.

Gene mutation analysis in the high- and low-risk groups. Abnormally gene mutation is one the main reasons resulting in tumorigenesis and tumor progression ${ }^{15,16}$. Multivariate regression analysis showed that there were four somatic mutations among those seven genes, including missense mutation, truncating mutation, amplification and deep deletion (Fig. 6A). To study the differences in gene mutations between the high- and low-risk groups, somatic processes were downloaded from the GDC database. The high- and low-risk samples in the file were respectively proposed and processed by MAftools $\mathrm{R}$ package. The top ten genes with higher mutation frequency in the high-risk group were TP53, APC, TTN, KRAS, SYNE1, MUC16, PIK3CA, FAT4, CSMD1 and DNAH11 (Fig. 6B). While, APC, TP53, TTN, KRAS, MUC16, PIK3CA, SYNE1, FAT4, SYR2 and OBSCN were the top 10 genes frequently mutated in the low-risk group (Fig. 6C). Next, in order to study the difference in mutation frequency of genes in the high- and low-risk groups, we used Fisher's test to analyze the frequency of mutations in each gene in the high- and low-risk groups, respectively, resulting in a total of 539 genes significantly mutated. The top 10 genes were MUC16, FAT4, BDP1, CMYA5, ZNF735, CENPE, SLITRK2, ZNF208, CFAP46 and ALDH1A2, as shown in Fig. $6 \mathrm{D}(\mathrm{p}<0.05)$. However, whether these gene mutations have biological function in CRC development or not should be validated by in vitro and in vivo experiments. 
Construction of the pharmacological network. To further explore the clinical value of the seven-gene prognostic MET biomarkers, DGIdb and TCMSP database were used to search for potential traditional Chinese herbs that may be used in the treatment of MET-mediated colorectal cancer patients. Firstly, 244 compounds were predicted to interact with AQP8, HPGD, CPT2 and CLCA1 by DGIdb. Thereafter, those 244 compounds were input into the TCMSP database, and a total of 115 herbs corresponding to 38 compounds, which interact with HPGD were identified. Thus, a pharmacological network composed of HPGD, 38 compounds and 115 herbs were constructed by Cytoscape software (Fig. 7).

\section{Discussion}

Despite targeted therapy and immunotherapy have brought significant clinical benefits to patients with advanced CRC. The five-year overall survival of CRC remains unsatisfactory, which is less than $30 \%{ }^{17}$. It is urgent to find more prognostic biomarkers and therapeutic agents to improve the outcomes of CRC patients. Emerging evidences have proposed that the abnormal metabolic genetic profile is closely involved in tumorigenesis. Some of these aberrant metabolites are significantly associated with the proliferation, progression, recurrence, and metastasis of cancer cells.

In the current study, we identified a total of 326 DEMET between CRC and normal tissues, which were mainly involved in various transmembrane transporter activity, metabolic processes, and drug metabolism. As known, Wnt/ $\beta$-catenin is the most important signaling pathway in regulating the initiation and development of $\mathrm{CRC}^{18}$. Interestingly, Wnt also increases glucose metabolism by directly regulating the transcriptional activity of pyruvate dehydrogenase knase 1 (PDK1) and lactate transporter, MCT-1 $(\mathrm{SLC16A1})^{19}$. In addition, as the most common and important genetic drivers of CRC, KRAS and p53 were all involved in cancer metabolism ${ }^{20,12}$ Therefore, metabolic process is seemed as one of the key hallmarks of CRC.

Importantly, in our study, a risk score model and a nomogram with high accuracy in predicting the prognosis of CRC patients were constructed based on 7-gene MET signature, including GDPD3, AQP8, GPX3, HPGD, CKMT2, CPT2 and CLCA1. GDPD3 is reported to play an essential role in maintaining stem cell quiescence and TKI resistance in chronic myeloid leukemia $(C M L)$ stem cells ${ }^{21}$. Specific inhibition of GDPD3-mediated lysophospholipid metabolism may be a viable manner of therapeutically targeting CML stem cells. However, the function and mechanism of GDPD3 in CRC is still unknown. Wu et al reported that AQP8 was downregulated in CRC, and its overexpression significantly decreased cell growth, colony formation and metastasis ${ }^{22}$. Zhang et al found that high level of AQP8 was significantly associated with better survival in CRC patients ${ }^{23}$. It looks like that AQP8 might be an independent indicator of poor prognosis in patients with CRC. GPX3 plays an important role in regulating the resistance of CRC cells to platinums, which are used in the first-line chemotherapy for $\mathrm{CRC}^{24}$. Hydroxyprostaglandin dehydrogenase 15-(nicotinamide adenine dinucleotide) (15-PGDH, HPGD) is down-regulated in CRC and associated with $\mathrm{CRC}$ risk through regulating a metabolic antagonist of $\mathrm{PTGS} 2^{25}$. Using univariate $\mathrm{COX}$ regression analysis, Liang et al identified five genes (PHACTR3, CKMT2, CYP2W1, ERFE, HOXC4) which were related 
to overall survival in left-sided colon cancer based on TCGA data ${ }^{26} .13$ potential prognostics differentially expressed metabolism-related genes (PDEMRGs) were identified in GC patients. One of them is CKMT2 ${ }^{27}$. Whereas, little is known about the expression and function of CKMT2 in CRC or GC. CPT2 expression was significantly downregulated in CRC patients, and its lower expression could induce stemness and oxaliplatin resistance by regulating ROS/Wnt/ $\beta$-catenin-induced glycolytic metabolism ${ }^{28}$. As for CLCA1, it has been reported to be downregulated and play multiple roles in CRC. Overexpression of CLCA1 inhibits the growth, proliferation and metastasis of CRC cells ${ }^{29}$. Wei et al found that CLCA1 is a potential diagnostic and prognostic marker for $\mathrm{CRC}$, and has a close relationship with tumor metabolism and immune invasion of $\mathrm{CRC}^{30}$. Those above findings indicates that GDPD3, AQP8, GPX3, HPGD, CKMT2, CPT2 and CLCA1 are potential therapeutic targets for some types of malignant tumors.

We then used GSEA analysis to evaluate the underlying mechanisms of prognostic MET biomarkers in $\mathrm{CRC}$, and found that the expressions of more than half of different kinds of $\mathrm{T}$ or $\mathrm{B}$ cells were significantly different between the high- and low-risk group. Especially, HPGD was strongly positively correlated with 26 kinds of immune cells and GPX3 had strong positive correlation with 23 kinds of immune cells. Both T cells and B cells have been shown to be important parts of the immune cell infiltrate of many tumor entities. Saleh $\mathrm{R}$ et al found that tumor-infiltrating $\mathrm{CD} 4+\mathrm{T}$ cells upregulated programmed cell death protein-1 (PD-1), cytotoxic T-lymphocyte-associated protein-4 (CTLA-4), T cell immunoglobulin and mucin domain-3 (TIM-3) and lymphocyte-activation gene 3 (LAG-3) ${ }^{31}$. Importantly, different $T$ cell subsets with distinct functions. Certain T cell subsets could be biomarkers of CRC patients with immunotherapy or CAR-T treatment ${ }^{32-34}$. Recently, ipilimumab (anti-CTLA-4) plus nivolumab (anti-PD-1) was approved for treatment of metastatic melanoma, advanced renal cell carcinoma and $\mathrm{mCRC}$ with MMR/MSI-H aberrations. In our study, the seven-gene high-risk group was more likely to be more sensitive to CTLA4 therapy. These findings increase our knowledge about the potential use of checkpoint blockade in CRC patients.

There are some limitations in the current study that the expressions of prognostic MET biomarkers are not validated in vivo, and further in vivo and in vitro experiments are required to unveil the mechanisms of them in regulating CRC. In addition, their clinical application also should be evaluated in the real-word study or our daily clinical practice. In conclusion, we identified seven METs that were significantly associated with prognosis in CRC, explored the underlying molecular mechanisms, and established the risk model and nomogram that could accurately predict prognosis of CRC patients. These models might facilitate the evaluation of prognosis and identification of patients with CRC who are more suitable for immunotherapy.

\section{Methods}

Data source. Gene expression profile and clinical information of $622 \mathrm{CRC}$ patients were downloaded from TCGA database. All methods were performed in accordance with the relevant guidelines and regulations ${ }^{35}$. When constructing the risk score model, CRC patients were randomly assigned to the 
training set and internal validation set at the ratio of 7:3. In addition, an external validation set containing 584 CRC patients was downloaded from GSE39582 containing 443 colon cancer and 19 non-tumoral colorectal mucosas. 2,752 METs were collected from the comprehensive gene list of human METs compiled by Richard Possemato et al as previously published ${ }^{36}$.

Identification and functional analysis of immune-related DERBPs. Limma package was used to identify DEGs between CRC and control samples with the threshold $\mid$ log2 (fold change) $\mid>1$ and $p$-value $<0.05$. Differentially expressed MET (DEMET) were obtained by overlapping DEGs with MET. Gene ontology (GO) and Kyoto encyclopedia of genes and genomes (KEGG) enrichment analysis of DEMET were performed using clusterProfiler package.

Construction of the risk model and nomogram based on prognostic signature. Univariate Cox regression was used to screen DEMET significantly associated with prognosis $(p<0.05)$. To get more robust prognostic signature, multivariate cox regression was performed to further filter DEMET from univariate cox regression analysis. The risk score of each patient was calculated as the following formula:

ExpGene $1{ }^{*}$ Coef $1+$ ExpGene2*Coef $2+$ ExpGene $3{ }^{*}$ Coef $3+\ldots+$ ExpGene $7{ }^{*}$ Coef 7

where Coef means the regression coefficients of genes, Exp is the normalized expression values of each prognostic MET signature. According to the median value of the risk score, CRC patients in the TCGA training set were divided into low- and high-risk groups. GO terms and KEGG pathways significantly enriched in low- and high-risk groups were analyzed by GSEA $(p<0.05)$. The overall survival $(O S)$ of lowand high-risk groups was analyzed by Kaplan-Meier. ROC curves were plotted to evaluate the performance of the risk model using the R package "survivalROC". The risk model was tested in both internal and external validation sets. Thereafter, univariate and multivariate Cox regression analyses were carried out to determine independent prognostic factors for CRC patients. Based on independent prognostic factors, a nomogram for predicting 1-, 3- and 5-year survival of CRC patients was established. The performance of the nomogram was evaluated by the calibration curves.

Evaluation of tumor immune microenvironment in low- and high-risk groups. To evaluate the tumor immune microenvironment in low- and high-risk groups, the enrichment levels of 28 immune cells, including activated B cell, activated CD4 T cell, activated CD8 T cell, activated dendritic cell, CD56bright natural killer cell, CD56dim natural killer cell, central memory CD4 T cell, central memory CD8 T cell, effector memory CD4 T cell, effector memory CD8 T cell, eosinophil, gamma delta T cell, immature B cell, immature dendritic cell, macrophage, mast cell, MDSC, memory B cell, monocyte, natural killer cell, natural killer T cell, neutrophil, plasmacytoid dendritic cell, regulatory T cell, $T$ follicular helper cell, type 1 T helper cell, type $17 \mathrm{~T}$ helper cell and type $2 \mathrm{~T}$ helper cell in each sample were calculated by ssGSEA. In addition, the expressions of immune checkpoints, including CD27, CD274, HAVCR2, ICOS, ID01, LAG3, PDCD1, PDCD1LG2 and TIGIT, were analyzed and compared between low- and high-risk groups.

Moreover, the response of patients in low- and high-risk groups to immunotherapy was predicted by TIDE algorithm. 
Tumor mutation analyze in the high- and low-risk groups. Tumor mutation data of CRC patients were downloaded from GDC database (https://portal.gdc.cancer.gov/), and the maftools R package was used to analyze the mutation data in both the high- and low-risk groups. Tumor mutation burden was calculated using the tumor-specific mutation genes. After that, the top 20 mutation genes in high- and low-risk group were listed. Fisher test was performed to compare the mutational frequencies of genes between low- and high-risk group. The forest plot was used to visualize the top 20 differentially mutated genes between low- and high-risk group. In addition, tumor heterogeneity of patients in low- and high-risk groups were analzyed by MATH algorithm in the maftools $\mathrm{R}$ package and compared by Wilcoxon test. A $p$-value $<0.05$ was considered as statistically significant difference between low- and high-risk group.

Construction of the pharmacological network. DGIdb database was used to screen active compounds that may interact with prognostic MET. Then the selected drugs were input into TCMSP database to further find matched herbs. Accordingly, the pharmacological network was constructed and visualized by Cytoscape software.

Statistical analysis. All data were analyzed by R (version 4.0.0). Wilcoxon test was used to compare the data between two groups. The chi-square test was used to compare the distribution of clinical features between low- and high-risk groups. Significant difference was considered as $p<0.05$ unless specified.

\section{Declarations}

\section{Acknowledgement}

This research was funded by grants from Yunnan Health Training Project of High-Level Talents (2018HB049, YNWR-QNBJ-2019-147, D-2017002), grant from the National Natural Science Foundation of China (81860509), and grant from Internal Division of Yunnan Province Health Commission (2018NS0265, 2018NS0266, 2016NS226).

\section{Author contributions}

Conceptualisation: Z.W.J, Z.Y.; Methodology: L.D.M. and C.M.; Formal analysis: Z.W.J., C.X.L.; Writing: Z.W.J., Z.Y.; Supervision: Z.W.J., Z.Y.

\section{Data availability statement}

Data and materials from this study are available upon a written request.

\section{A Competing Interests Statement}

The authors declare no competing interests.

\section{References}


1. Zhou, M., et al. Mortality, morbidity, and risk factors in China and its provinces, 1990-2017: a systematic analysis for the Global Burden of Disease Study 2017. Lancet. 394, 1145-1158 (2019).

2. Piawah, S., Venook, A.P. Targeted therapy for colorectal cancer metastases: A review of current methods of molecularly targeted therapy and the use of tumor biomarkers in the treatment of metastatic colorectal cancer. Cancer. 125, 4139-4147 (2019).

3. Engstrand, J., Nilsson, H., Strömberg, C., Jonas, E. \& Freedman, J. Colorectal cancer liver metastases a population-based study on incidence, management and survival. BMC Cancer. 18,78 (2018).

4. Pavlova, N.N., Thompson, C.B. The Emerging Hallmarks of Cancer Metabolism. Cell Metab. 23, 2747 (2016).

5. Ward, P.S., Thompson, C.B. Metabolic reprogramming: a cancer hallmark even warburg did not anticipate. Cancer Cell. 21, 297-308 (2012).

6. Bott, A.J., et al. Glutamine Anabolism Plays a Critical Role in Pancreatic Cancer by Coupling Carbon and Nitrogen Metabolism. Cell Rep. 29, 1287-1298.e6 (2019).

7. Chen, L.Q., Cheung, L.S., Feng, L., Tanner, W., Frommer, W.B. Transport of sugars. Annu Rev Biochem. 84, 865-94 (2015).

8. Matés, J.M., Campos-Sandoval, J.A., Santos-Jiménez, J.L., Márquez, J. Dysregulation of glutaminase and glutamine synthetase in cancer. Cancer Lett. 467, 29-39 (2019).

9. Yang, D., Tu, R.F., Liu, H.D., Qing, G.L. Regulation of cancer cell metabolism: oncogenic MYC in the driver's seat. Sig Transduct Target Ther. 5, 124 (2020)

10. Samec, M., et al. Flavonoids Targeting HIF-1: Implications on Cancer Metabolism. Cancers (Basel). $13,130(2021)$

11. Gao, L., et al. CPI-613 rewires lipid metabolism to enhance pancreatic cancer apoptosis via the AMPK-ACC signaling. J Exp Clin Cancer Res. 39, 73 (2020).

12. Liu, J., Zhang, C., Hu, W., Feng, Z. Tumor suppressor p53 and metabolism. J Mol Cell Biol. 11, 284292 (2019).

13. Yu, Y., Gong, L., Ye, J. The Role of Aberrant Metabolism in Cancer: Insights into the Interplay Between Cell Metabolic Reprogramming, Metabolic Syndrome, and Cancer. Front Oncol. 10, 942 (2020).

14. Harmon, C., O'Farrelly, C., Robinson, M.W. The Immune Consequences of Lactate in the Tumor Microenvironment. Adv Exp Med Biol. 1259, 113-124 (2020).

15. Raskov, H., Søby, J.H., Troelsen, J., Bojesen, R.D., Gögenur, I. Driver Gene Mutations and Epigenetics in Colorectal Cancer. Ann Surg. 271, 75-85 (2020).

16. Sun, S., Klebaner, F., Zhang, X., Tian, T. Instantaneous mutation rate in cancer initiation and progression. BMC Syst Biol. 12 (Suppl 6):110 (2018).

17. Mangone, L., et al. Five-year relative survival by stage of breast and colon cancers in Italy. Tumori. 107, 318-324 (2021).

18. Bian, J., Dannappel, M., Wan, C., Firestein, R. Transcriptional Regulation of Wnt/ $\beta$-Catenin Pathway in Colorectal Cancer. Cells. 9, 2125 (2020). 
19. Sprowl-Tanio, S., et al. Lactate/pyruvate transporter MCT-1 is a direct Wnt target that confers sensitivity to 3-bromopyruvate in colon cancer. Cancer Metab. 4, 20 (2016).

20. Rozeveld, C.N., Johnson, K.M., Zhang, L., Razidlo, G.L. KRAS Controls Pancreatic Cancer Cell Lipid Metabolism and Invasive Potential through the Lipase HSL. Cancer Res. 80, 4932-4945 (2020).

21. Naka, K., et al. The lysophospholipase D enzyme Gdpd3 is required to maintain chronic myelogenous leukaemia stem cells. Nat Commun. 11, 4681 (2020).

22. Wu, Q., et al. AQP8 inhibits colorectal cancer growth and metastasis by down-regulating PI3K/AKT signaling and PCDH7 expression. Am J Cancer Res. 8, 266-279 (2018).

23. Zhang, $\mathrm{H}$., et al. Identification of the AQP8-miR-92a network associated with the aggressive traits of colorectal cancer. Biochem Biophys Res Commun. 527, 218-225 (2020).

24. Pelosof, L., et al. GPX3 promoter methylation predicts platinum sensitivity in colorectal cancer. Epigenetics. 12, 540-550 (2017).

25. Fink, S.P., et al. Aspirin and the risk of colorectal cancer in relation to the expression of 15hydroxyprostaglandin dehydrogenase (HPGD). Sci Transl Med. 6, 233re2 (2014).

26. Liang, L., et al. Distinguishable Prognostic Signatures of Left- and Right-Sided Colon Cancer: a Study Based on Sequencing Data. Cell Physiol Biochem. 48, 475-490 (2018).

27. Wen, F., et al. Identification and prognostic value of metabolism-related genes in gastric cancer. Aging (Albany NY). 12, 17647-17661 (2020).

28. $\mathrm{Li}, \mathrm{H}$., et al. CPT2 downregulation triggers stemness and oxaliplatin resistance in colorectal cancer via activating the ROS/Wnt/ $\beta$-catenin-induced glycolytic metabolism. Exp Cell Res. 409, 112892 (2021).

29. Li, X., et al. CLCA1 suppresses colorectal cancer aggressiveness via inhibition of the Wnt/betacatenin signaling pathway. Cell Commun Signal. 15, 38 (2017).

30. Wei, F.Z., et al. Differential Expression Analysis Revealing CLCA1 to Be a Prognostic and Diagnostic Biomarker for Colorectal Cancer. Front Oncol. 10, 573295 (2020).

31. Saleh, R., Toor, S.M., Khalaf, S., Elkord, E. Breast Cancer Cells and PD-1/PD-L1 Blockade Upregulate the Expression of PD-1, CTLA-4, TIM-3 and LAG-3 Immune Checkpoints in CD $4+$ T Cells. Vaccines (Basel). 7, 149 (2019).

32. Zhang, L., et al. Lineage tracking reveals dynamic relationships of T cells in colorectal cancer. Nature. 564, 268-272 (2018).

33. Toor, S.M., et al. Immune Checkpoints in Circulating and Tumor-Infiltrating CD4 + T Cell Subsets in Colorectal Cancer Patients. Front Immunol. 10, 2936 (2017).

34. Feng, M., Zhao, Z., Yang, M., Ji, J., Zhu, D. T-cell-based immunotherapy in colorectal cancer. Cancer Lett. 498, 201-209 (2021).

35. Wang, Z., Jensen, M.A., Zenklusen, J.C. A Practical Guide to The Cancer Genome Atlas (TCGA). Methods Mol Biol. 1418, 111 - 41 (2016). 
36. Possemato, R., et al. Functional genomics reveal that the serine synthesis pathway is essential in breast cancer. Nature. 476, 346-50 (2011).

\section{Tables}

Tables 1 and 2 are only available as a download in the Supplemental Files section.

\section{Figures}

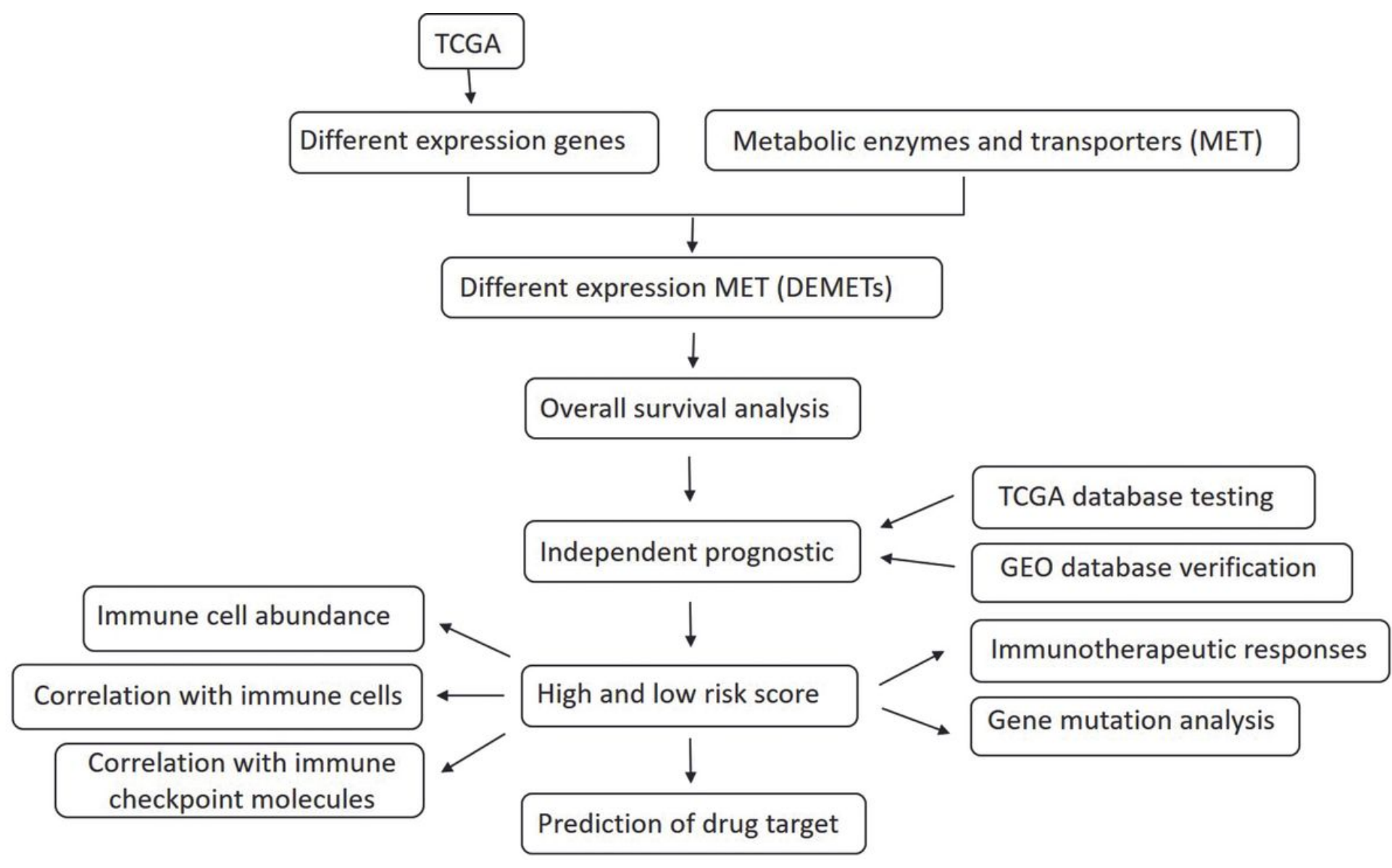

Figure 1

Schematic illustration of gene selection, data processing, and analysis of our study. 
a

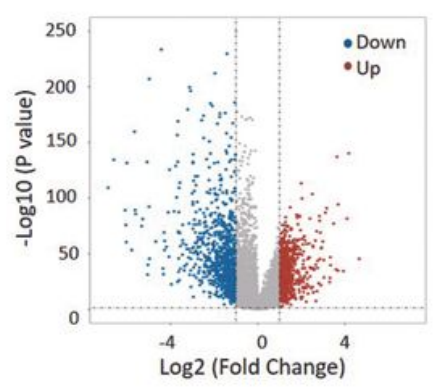

b

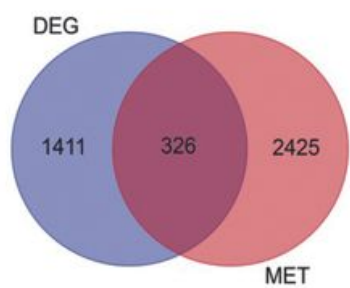

C

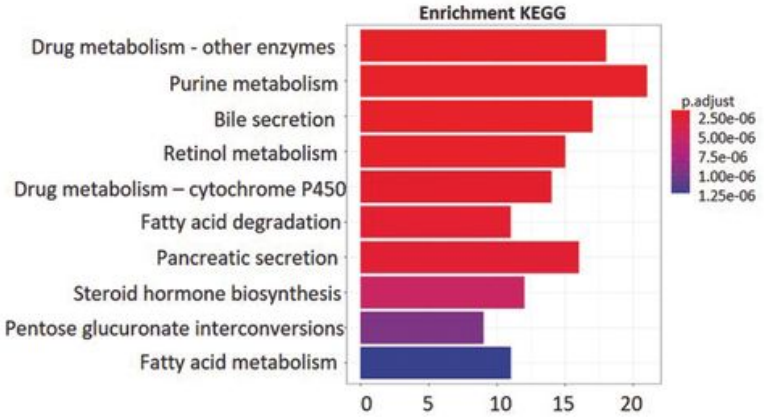

d

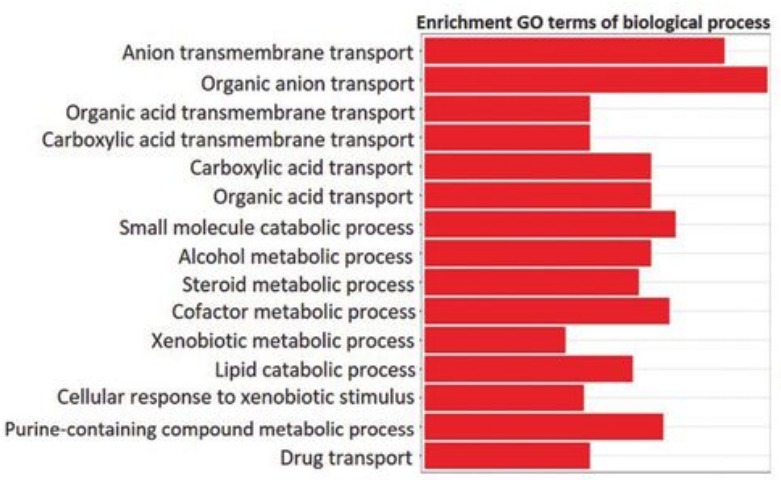

e

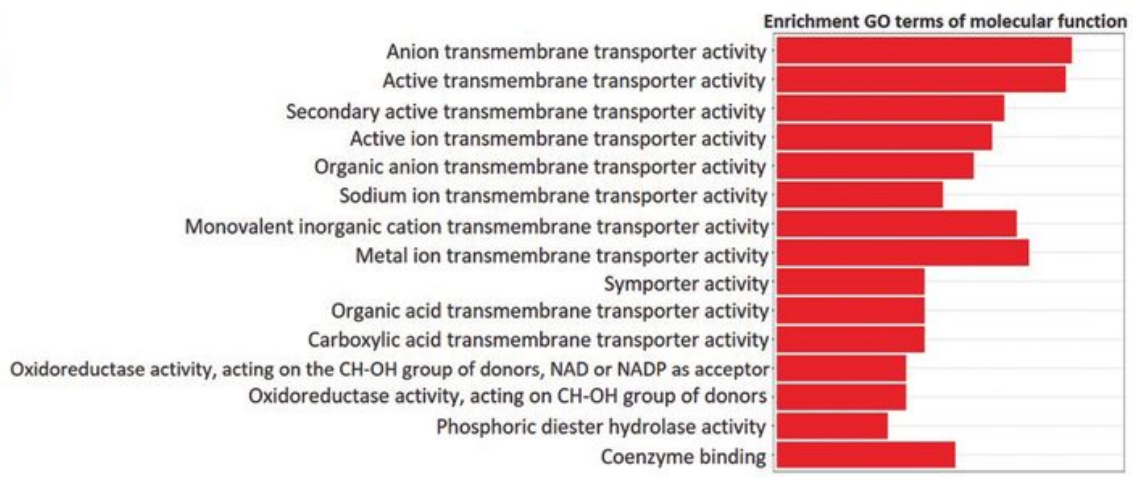

\section{Figure 2}

\section{Identification and functional analysis of 326 DEMET in CRC patients.}

(a) Volcano plot of differentially expressed genes (DEGs) between CRC and normal tissues. Blue indicates downregulated DEGs, red indicates upregulated DEG, and gray indicates genes that were not significantly differentially expressed. (b) The Venn plot of 1,737 DEGs and 2,752 MET. (c) KEGG pathway enrichment analysis of DEGs. (d\&e) GO enrichment analysis and remarkably enhanced biological process and molecular function terms of DEGs. 


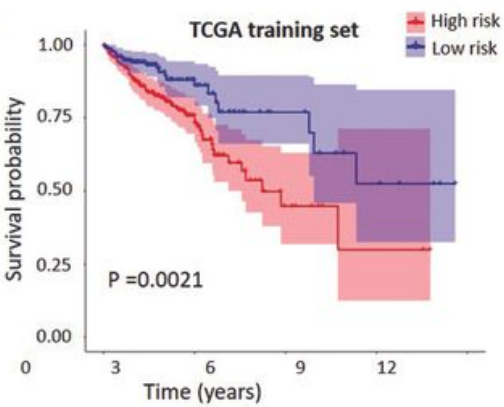

b

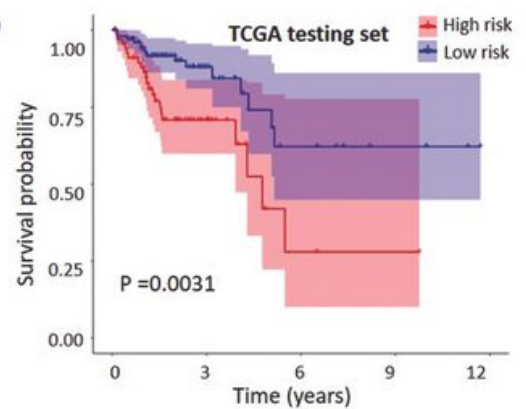

C

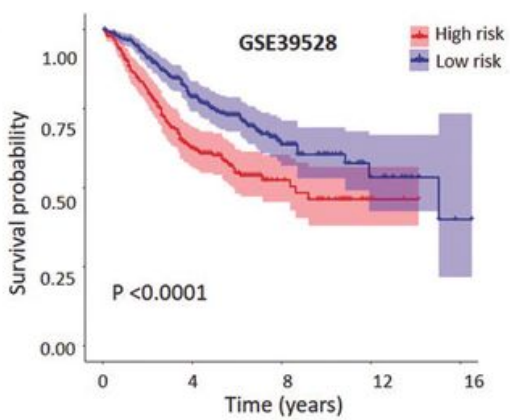

d

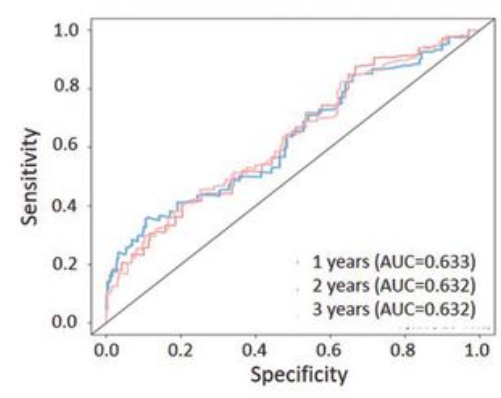

e

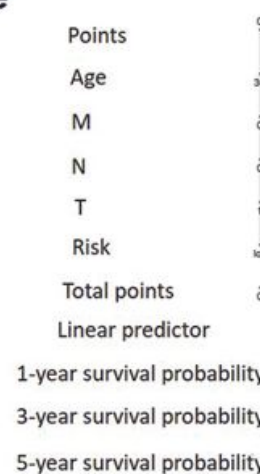

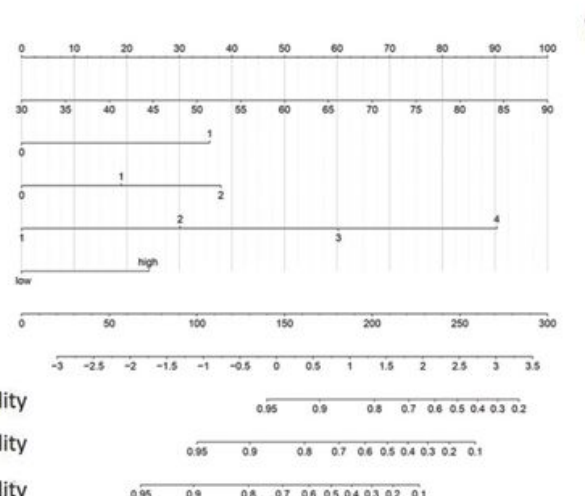

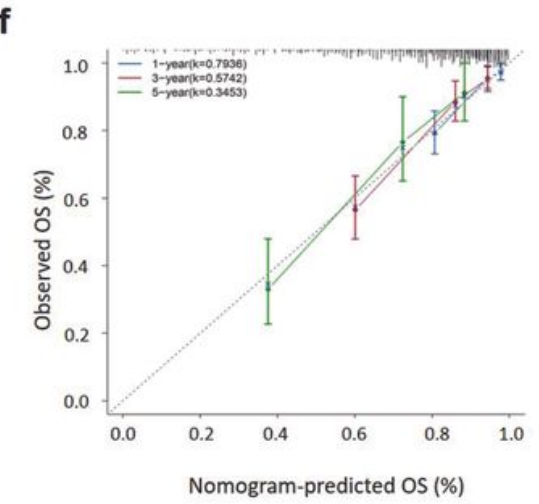

Figure 3

prognostic value of genetic risk score model and nomogram construction.

The Kaplan-Meier analysis for the survival of patients in low- and high-risk groups in the TCGA training set (a), testing set (b) and GSE39528 (c). (d) The survival time-dependent ROC curves of 1-, 3- and 5-year survival. AUC, area under the curve. ROC, receiver operating characteristic. (e) A nomogram to predict the survival based on independent prognostic factors. (f) Calibration curves of the nomogram for 1-, 3-, and 5-year.

a

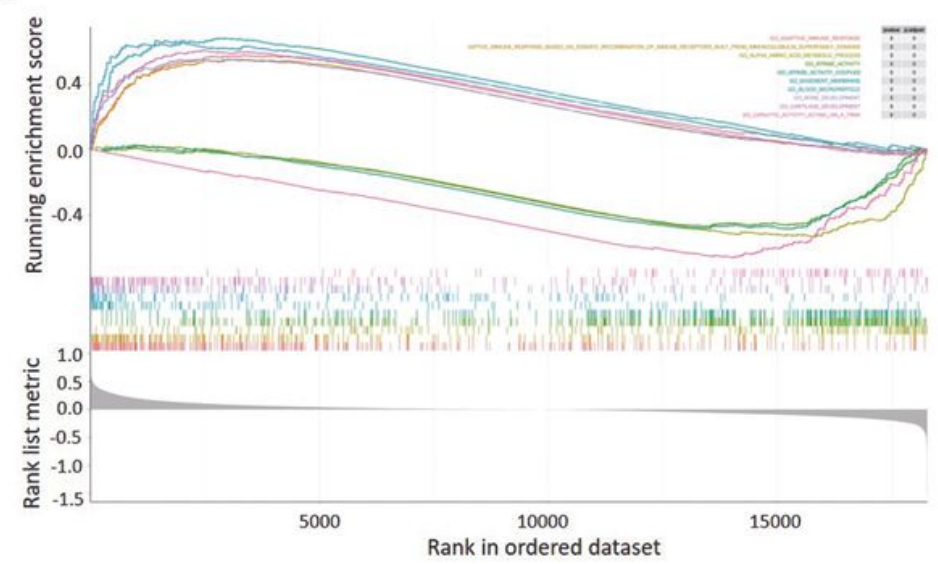

b

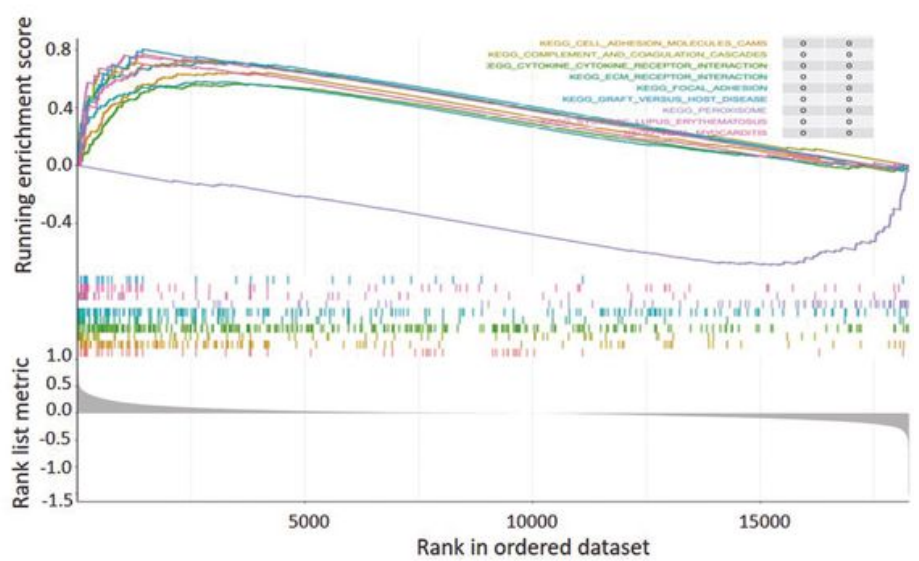

Figure 4

Significantly enriched KEGG pathways and GO terms in training cohort by GSEA. 
The top $10 \mathrm{GO}$ terms (a) and top 10 KEGG pathways (b). Above the horizontal axis indicated the pathways are significantly enriched in the high-risk group, and below the horizontal axis indicated that the pathways are significantly enriched in the low-risk group.

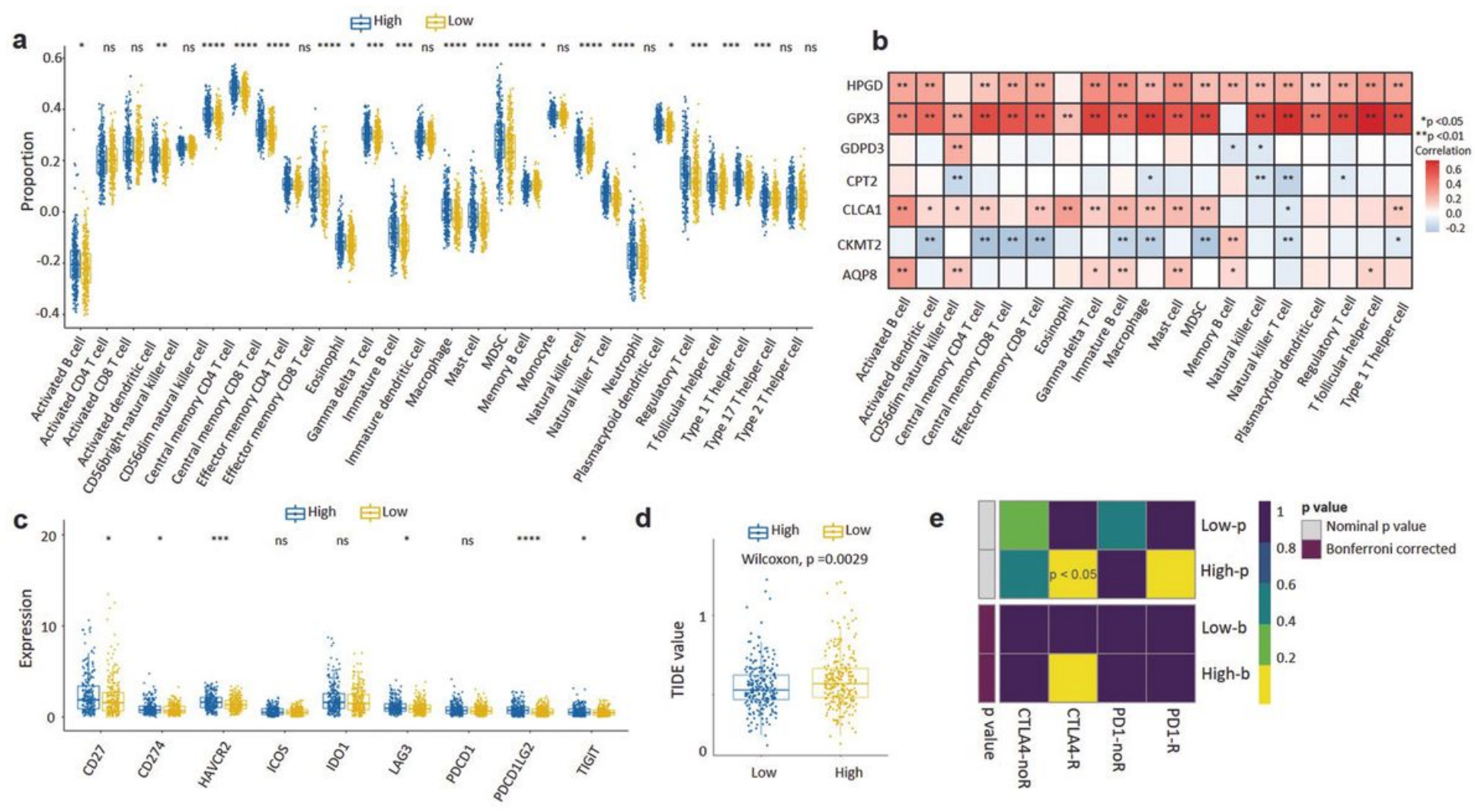

Figure 5

Different tumor immune microenvironment between low- and high-risk groups.

(a) Boxplots of sSGSEA inferred cell content between low- and high-risk group. (b) Heatmap of correlations between seven prognostic MET biomarkers and immune cells. Red represents positively correlation; blue represents negatively correlation. (c) Comparison of the expressions of immune checkpoints between low- and high-risk group. (d) Comparison of the response of patients to immunotherapy (TIDE score) between low- and high-risk group. (e) Response to immunotherapy in highand low-risk groups. 


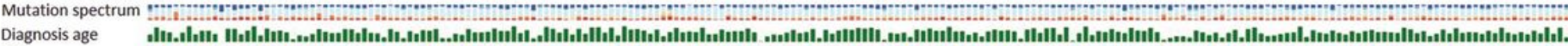

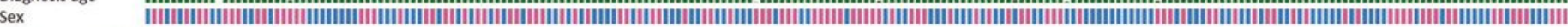

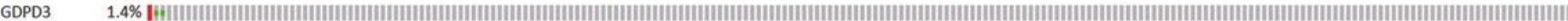

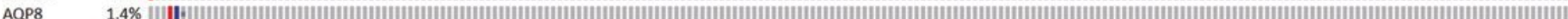

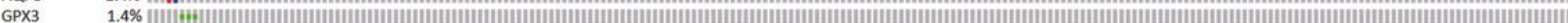

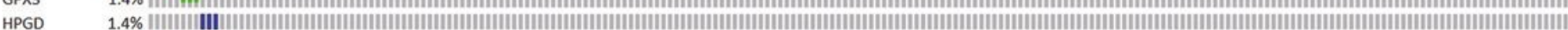

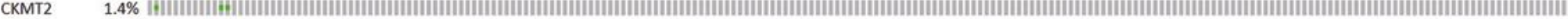

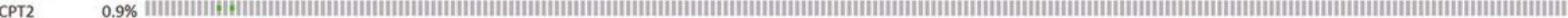

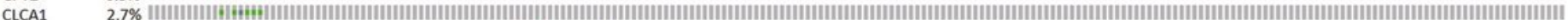

Genetic alteration

Mutation spectrum

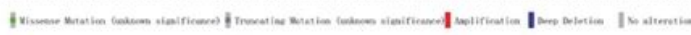

Diagnosis age

Ios los for los foc I

Sex
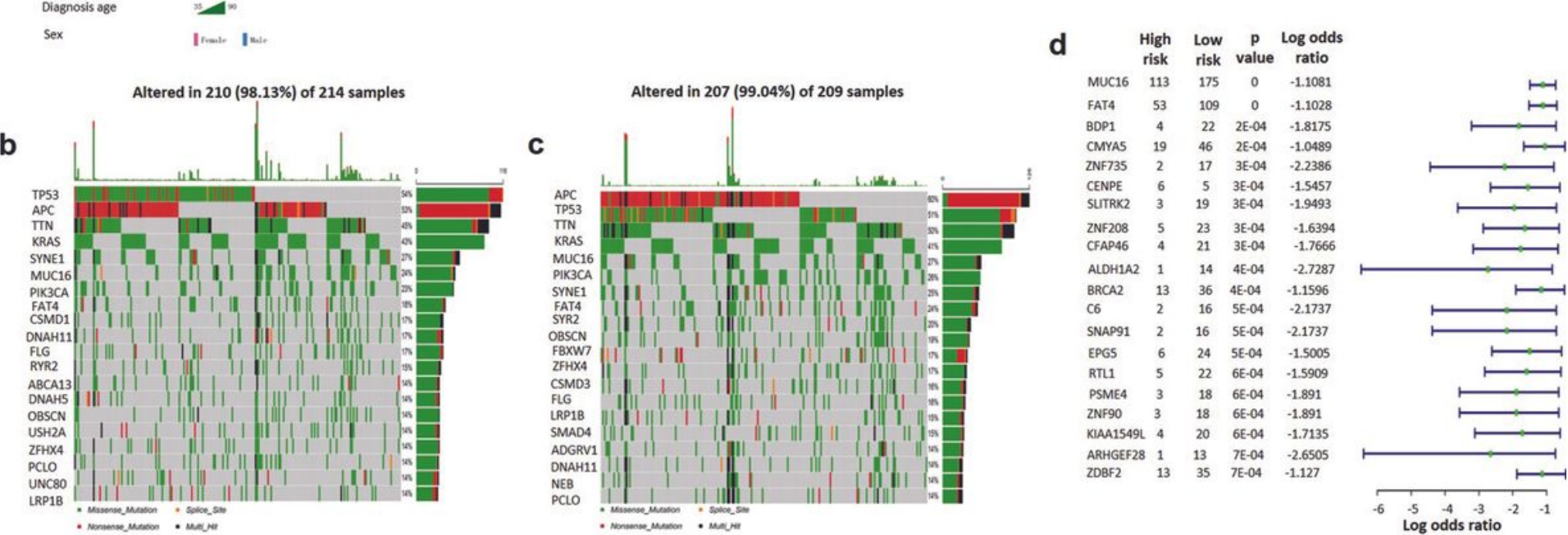

Figure 6

\section{Summary of gene mutation types}

(a)

Mutation spectrum represents the number of point mutations calculated by different types of nucleotide changes in a sample. Waterfall diagram of the top20 genes with the most mutations in highrisk samples (b) and low-risk samples (c). (d) Top 20 mutated-genes commonly present in both the highand low-risk groups. 


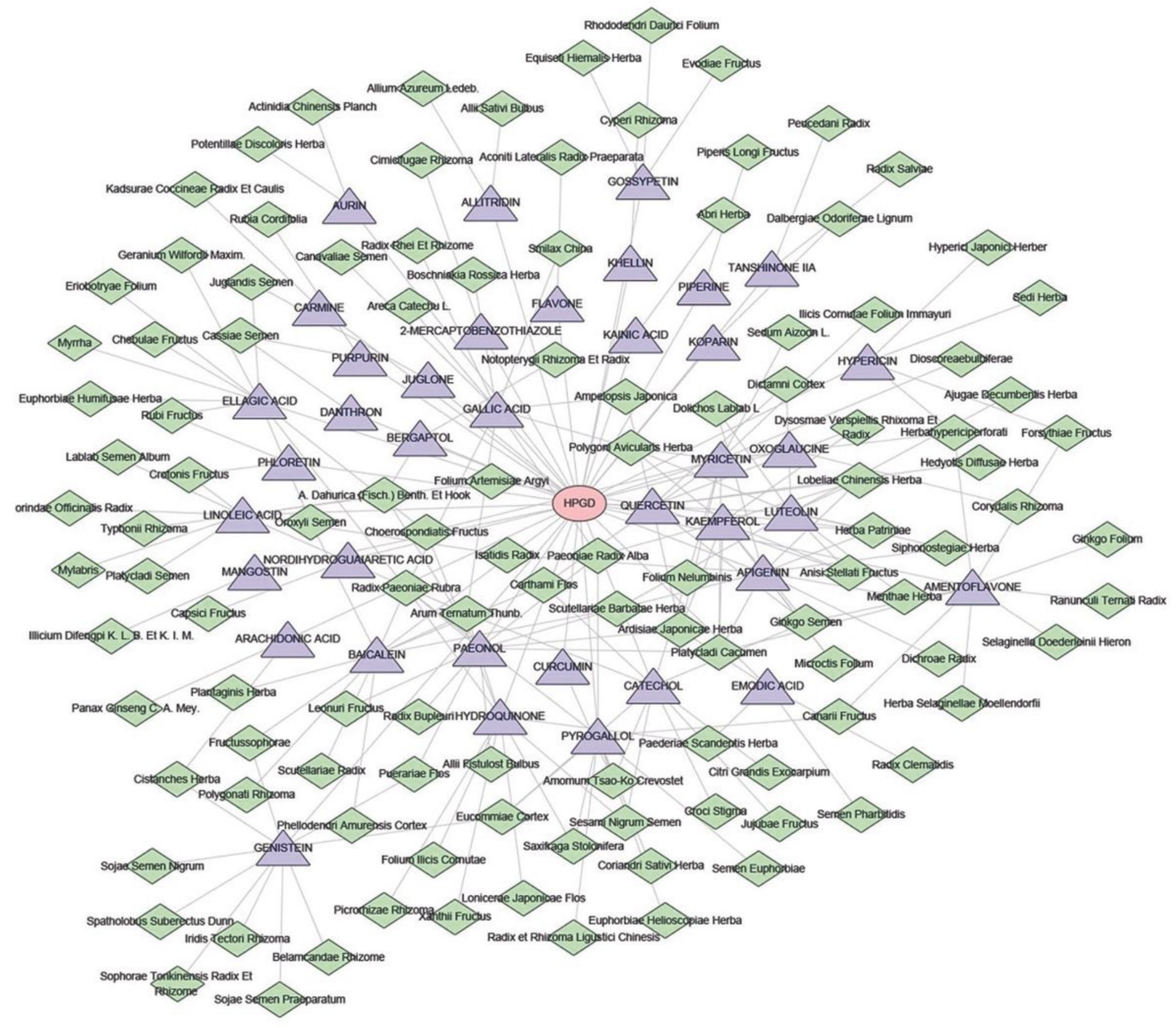

Figure 7

Construction of the pharmacological network

A pharmacological network composed of HPGD, 38 compounds and 115 herbs were constructed by Cytoscape software.

\section{Supplementary Files}

This is a list of supplementary files associated with this preprint. Click to download.

- table1.jpg 
- table2.jpg

Page 18/18 